\title{
Symptom Prevalence and Related Distress in Cancer Patients Undergoing Chemotherapy
}

\author{
Muthukkumaran Thiagarajan ${ }^{1}$, Caryn Mei Hsien Chan ${ }^{2 *}$, Ho Gwo Fuang ${ }^{1}$, Tan \\ Seng Beng ${ }^{2}$, MA Atiliyana ${ }^{1}$, NA Yahaya ${ }^{3}$
}

\begin{abstract}
Background: Much has been done to examine the psychological impact of cancer treatment, but it remains unclear to what extent anxiety and depression is related to symptom prevalence. The present study concerned the characteristics and frequency of distress as related to symptom prevalence in cancer patients undergoing chemotherapy in Malaysia. Materials and Methods: Participants were 303 consecutive adult cancer patients undergoing chemotherapy in an academic medical center. The short form Memorial Symptom Assessment Scale (MSAS-SF), which covers three domains of symptoms (global distress, physical- and psychological symptoms) was used to cross-sectionally measure symptom frequency and associated distress via self-reporting. One-way ANOVA and t-tests were used to test mean differences among MSAS-SF subscale scores. Results: Complete data were available for 303 patients. The mean number of symptoms was 14.5. The five most prevalent were fatigue, dry mouth, hair loss, drowsiness and lack of appetite. Overall, symptom burden and frequency were higher than in other published MSAS-SF studies. Higher symptom frequency was also found to be significantly related to greater distress in cancer patients undergoing chemotherapy. Conclusions: Patients undergoing chemotherapy suffer from multiple physical and psychological symptoms. Better symptom control or palliative care is needed. Greater frequency of reported symptoms may also indicate a subconscious bid by patients for care and reassurance - thus tailored intervention to manage distress should be offered.
\end{abstract}

Keywords: Cancer - chemotherapy - symptom prevalence - distress - psychosomatic effects

Asian Pac J Cancer Prev, 17 (1), 171-176

\section{Introduction}

Patients with cancer, particularly those undergoing treatment often experience multiple symptoms that cause significant distress and impair day-to-day functioning (Spichiger et al., 2011). Cytotoxic chemotherapy remains a mainstay in management of variety of malignancies, be it in the adjuvant or palliative setting. Although chemotherapy improves cancer survivorship, the side effects of these agents frequently render significant physical and psychological sequelae (Portenoy et al., 1994a).

A symptom is defined as a subjective phenomenon regarded by the individual as an indication or characteristic departing from normal function, sensation or appearance (Rhodes and Watson, 1987). Symptom distress refers to the degree of physical or mental suffering, discomfort or bother reported by individuals in relation to their perception of the symptom (Rhodes and Watson, 1987). Although symptoms experienced generally depend on treatment, primary tumour site or other co-morbid conditions, certain symptoms may be more closely related to psychological reasons (Cleeland, 2007). Multiple symptoms may also occur as a cluster (Illi, Miaskowski, Cooper et al., 2012).

While numerous studies have reported the occurrence of symptoms among cancer patients undergoing chemotherapy (Cooley et al., 2003; Ferguson and Ahles, 2003; Stasi et al., 2003; Kim et al., 2009), less is known about psychological symptoms and distress arising in apropos to symptom prevalence. Although these studies are heterogeneous in terms of patient population and assessment tool, the observed number of concurrent symptoms is consistently high (Portenoy et al., 1994b; Kim et al., 2009; Manitta et al., 2011; Petterson 2014). This observation is of great importance as a higher incidence of symptoms relates to poor compliance to chemotherapy (Stasi et al., 2003).

Treatment-related symptoms caused by treatment may be severe enough to cause intercycle delays during chemotherapy or lead to patients prematurely terminating treatment, while residual symptoms caused by chemotherapy often complicates post-treatment recovery and pose a hindrance to resumption of pre-disease levels 
of functioning (Cleeland, 2007). Higher symptom distress is also associated with poorer cancer-related survival (Cooley et al., 2003). Indeed, physical and psychological symptoms often converge and present in clusters. It is estimated that a third of cancer patients undergoing chemotherapy experience decline in cognitive function which affects their quality of life which may persist long after discontinuation of treatment (Ferguson and Ahles, 2003).

Given the widely recognised impairments of multiple symptoms, there is a need to evaluate the prevalence and impact of the symptoms and its associated distress in cancer patients undergoing active treatment in routine care, particularly as patients are surviving cancer for increasingly longer periods of time. These types of evaluation could potentially be used to guide the development and testing of interventions for multiple symptoms (Kim et al, 2009). There is also a need to assess the degree to which symptom frequency is related to distress.

This study was thus carried out to assess the prevalence of symptoms and its associated distress in cancer patients undergoing chemotherapy. The secondary aim was to identify characteristics of patient subgroups that are more likely to report multiple symptoms and/or distress in conjunction with chemotherapy.

\section{Materials and Methods}

\section{Participants}

Participants comprised of adult cancer patients undergoing chemotherapy at the oncology clinic in a single academic medical center. All patients undergoing any chemotherapeutic regime as inpatients or outpatients during the study period from 1 December 2009 to 31 March 2010 were included. The single inclusion criteria was being over 18 years of age. Patients who were undergoing concurrent radiotherapy were excluded from participating. Institutional Ethics Committee approval was obtained for this study.

\section{Materials}

The short form Memorial Symptom Assessment Scale (MSAS-SF) is a patient-rated, multidimensional instrument that evaluates the intensity, frequency, and distress associated with symptoms. This assessment tool was chosen due to its previously demonstrated excellent association with clinical status and quality of life measures, in addition to being a relatively easy tool for patients to complete (Chang et al., 2000a). The MSAS-SF yields several validated subscale scores, including a Global Distress Index (GDI), a Physical Symptom subscale score (MSAS-PHYS), and a Psychological Symptom subscale score (MSAS-PSYCH). Demographic information which included age, gender, ethnicity, diagnosis by site and diagnosis by stage were additionally collected.

\section{Procedure}

This was a cross-sectional survey study using consecutive sampling. Patients attending outpatient consultations in the waiting room of the oncology clinic or whom were receiving chemotherapy as inpatients were requested to complete a self-administered Memorial Symptom Assessment Scale Short Form (MSAS-SF) in English or Chinese. Patients who were unable to read either language were assisted by one of the researcher who helped fill the short form by interview.

\section{Design, data analyses and variables}

All analyses were performed using SPSS (v.15). Subgroup analysis and difference between the means was determined using student t-test and one-way analysis of variance. A level of significance of $p<0.05$ was considered significant.

\section{Results}

Out of 325 patients who underwent chemotherapy between December 2009 and March 2010,303 responded to the questionnaire. A total of 22 patients were not keen on taking part in this study, which gave a response rate of $93 \%$.

The mean age $( \pm \mathrm{SD})$ of the patients was 52.7 years $( \pm 12.1)$. The majority of patients were female $(75.2 \%)$, with breast or colorectal cancer $(65.1 \%)$. All of the patients $(100 \%)$ received chemotherapy. Five patients $(1.7 \%)$ had undergone radiotherapy at the point of the study. Although most of them had advanced cancer (stage III and IV cancer) $(69.3 \%)$, the majority (72.6\%) had ECOG performance status scores of less than 2 (Table 1).

Patients with poorer performance status as well as those who were receiving in-patient chemotherapy at the time of the study were more likely to report experiencing

Table 1. Sociodemograhic and Clinical Characteristics of Cancer Patients $(\mathrm{N}=\mathbf{3 0 3})$

\begin{tabular}{lrl}
\hline Characteristics & $\mathrm{N}(\%)$ \\
\hline Gender & $75(24.8)$ \\
Male & 228 & $(75.2)$ \\
Female & & \\
Ethnicity & 73 & $(24.1)$ \\
Malay & 193 & $(63.7)$ \\
Chinese & 32 & $(10.6)$ \\
Indian & 5 & $(1.70)$ \\
Others & & \\
ECOG Performance Status & 94 & $(31.0)$ \\
0 & 135 & $(41.6)$ \\
1 & 51 & $(16.8)$ \\
2 & 23 & $(7.60)$ \\
3 & 0 & $(0.00)$ \\
4 & & \\
Stage of Cancer & 23 & $(7.60)$ \\
I & 70 & $(23.1)$ \\
II & 83 & $(27.4)$ \\
III & 127 & $(41.9)$ \\
IV & & \\
Cancer by Site & 139 & $(45.9)$ \\
Breast & 52 & $(17.2)$ \\
Colorectal & 22 & $(7.30)$ \\
Lung & 21 & $(6.90)$ \\
Head and Neck & 29 & $(9.60)$ \\
Gynaecological & 40 & $(13.2)$ \\
Others & & \\
\hline
\end{tabular}


Table 2. Descriptive Statistics by Cancer Patient Characteristics and Subscale $(\mathbf{N}=303)$.

\begin{tabular}{|c|c|c|c|c|c|}
\hline & Frequency & GDI & PHYS & PSYS & TMSAS \\
\hline All Patients $(\mathrm{n}=303)$ & 14.5 & 1.06 & 1.46 & 1.01 & 0.7 \\
\hline Range & $0-30$ & $0.00-3.40$ & $0.80-3.47$ & $0.00-3.83$ & $0.00-2.68$ \\
\hline \multicolumn{6}{|l|}{ Gender } \\
\hline Male $(\mathrm{n}=75)$ & 14 & 0.59 & 0.82 & 0.48 & 0.67 \\
\hline Female $(n=228)$ & 14.7 & 0.59 & 0.82 & 0.53 & 0.71 \\
\hline \multicolumn{6}{|l|}{ Ethnicity } \\
\hline Malay $(n=73)$ & 15.8 & 1.23 & 1.6 & 1.15 & 0.83 \\
\hline Chinese $(n=193)$ & 13.6 & 0.96 & 1.4 & 0.91 & 0.63 \\
\hline Indian $(\mathrm{n}=32)$ & 17.4 & 1.23 & 1.5 & 1.31 & 0.84 \\
\hline Others $(\mathrm{n}=5)$ & 14.4 & 0.96 & 1.44 & 1.03 & 0.61 \\
\hline \multicolumn{6}{|l|}{ ECOG Performance Status } \\
\hline Good $(0-1)(n=229)$ & 14 & 0.95 & 1.37 & 0.92 & 0.61 \\
\hline Poor $(2-3)(n=74)$ & 16.3 & 1.38 & 1.73 & 1.28 & 0.98 \\
\hline \multicolumn{6}{|l|}{ Stage } \\
\hline I, II \& III (Adjuvant) $(\mathrm{n}=176)$ & 14.5 & 1.02 & 1.38 & 1.04 & 0.66 \\
\hline IV (Palliative) $(\mathrm{n}=127)$ & 14.6 & 1.11 & 1.56 & 0.97 & 0.76 \\
\hline \multicolumn{6}{|l|}{ Chemotherapy setting } \\
\hline In-patient $(\mathrm{n}=35)$ & 17.4 & 0.75 & 1.17 & 0.7 & 0.99 \\
\hline Out-patient $(n=268)$ & 14.1 & 0.57 & 0.78 & 0.49 & 0.67 \\
\hline
\end{tabular}

GDI - Global Distress Index; PHSY - Physical Symptom Subscale Score; PSYS - Psychological Symptom Subscale Score; TMSAS - Total Memorial Symptom Assessment Scale

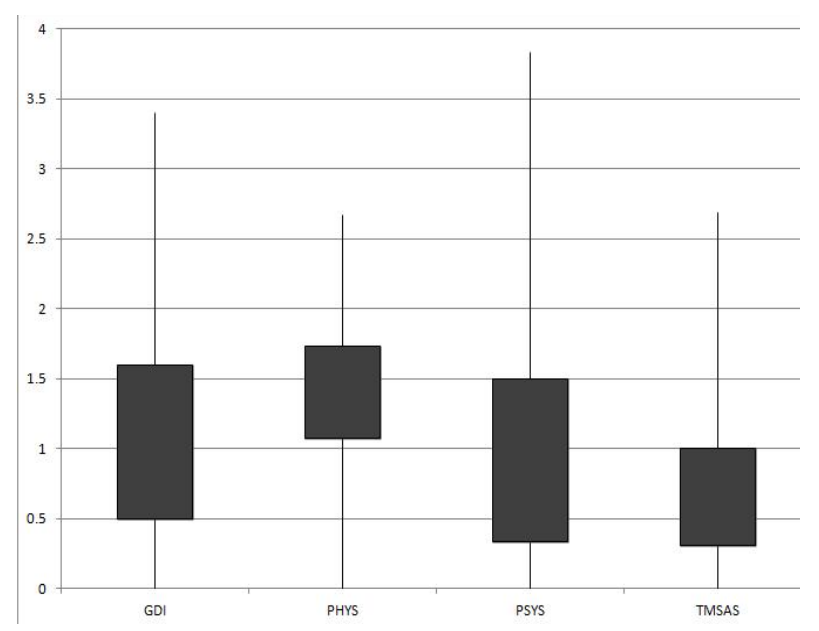

Figure 1. Boxplot of MSAS-SF Scores (as means) by Aubscales. Boxes represent lower and upper quartiles, the lines represent the most extreme values within $5 \%$ range of the quartile ends

more symptoms. These patients were also more likely to be distressed by their symptoms as demonstrated in Table 2 .

The average number of symptoms reported by patients by mean frequency or symptom burden was $14.5 \pm 6.4$. The highest variation in score was seen in the psychological symptom subscale as depicted in Figure 1, in comparison to the other subscales.

As illustrated in Figure 2, the most common physical symptoms were the lack of energy, dry mouth, hair loss, drowsiness and lack of appetite, as reported by more than $60 \%$ of patients. Swelling of limbs, sexual disturbances, urinary and swallowing difficulties as well as itching were the least prevalent, with each of these reported by less than $30 \%$ of patients.

Lack of energy and lack of appetite were not just among the most prevalent symptoms, but the patients who reported these symptoms were more distressed by them compared to other symptoms. Other symptoms such as

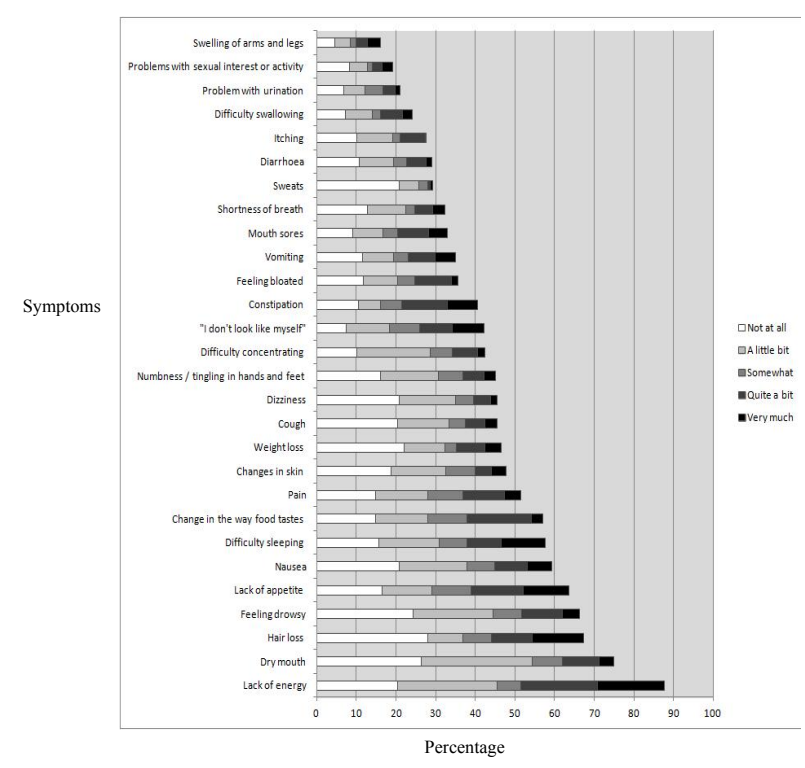

Figure 2. Frequency and Severity (in percentage) of Chemotherapy Related Symptoms Experienced by Patients

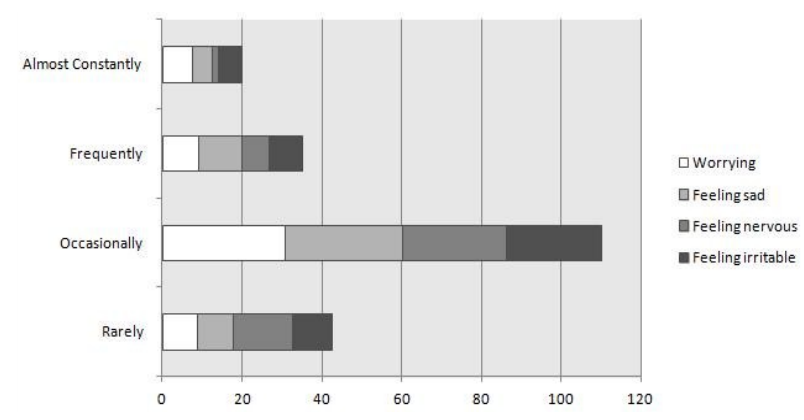

Figure 3. Frequency of Psychological Symptoms among Cancer Patients as Measured Using the MSAS-SF Psychological Subscale

constipation, pain and "I don't look like myself" were prevalent in about half of the patients. Most of the patients 
who complained of these symptoms reported moderate to severe distress in association with these symptoms.

Physical symptoms were more highly reported compared to psychological symptoms, while the latter was generally occasional in frequency. The median for all four subscale scores measuring mood was below 2.0. A large range of these scores showed a wide score variation among the values.

\section{Discussion}

The present study offers rare findings from a Southeast Asian setting examining a heterogeneous group of patients in terms of cancer by type and stage. Consistent with the most recent findings, no differences were identified in terms of disease and treatment characteristics (Miaskowsi et al., 2014). The symptom burden in this study population was greater compared to published data (Zabora et al., 2001).

A marked incongruity was observed between the number and frequency of physical symptoms reported with the associated psychological distress reported. Patients with advanced disease had significantly higher physical subscale scores, but not necessarily greater psychological distress.This was surprising as a higher level of reported distress would have been expected given the high number of physical symptoms reported. This may be attributable to barriers to reporting symptoms particularly given the fact that Asian patients do not typically express distress unless elicited (Borjeson, Starkhammar, Unosson, Bertero, 2012).

Distress that was indeed reported was, interestingly, clustered to particular symptoms. The most distressing symptoms were the lack of energy, lack of appetite and change in the way food tastes. Pain, however, which was reported by half of the study population, was less reported to cause distress. Only half of patients who reported pain reported being moderately to severely distressed by it, in stark contrast to prior research findings (Breen et al., 2009).

As indicated by previous research in western settings, patients rarely express psychological distress and concerns directly (Uitterhoeve et al., 2009; Delgado-Guay et al., 2009; Heyn et al., 2012). It may be possible that the more frequent expression of multiple symptoms could perhaps indicate a latent expression of psychological distress. This concern is consistent with and may hold particularly true in the Asian culture, where somatisation is generally more common (Akechi et al., 2011; Huijer et al., 2012).

Another striking finding of this study was that despite nausea and vomiting being prevalent symptoms in this study, they were not reported to be highly distressing. This was at odds with the literature and findings such as by Sun et al. (2005) who reported nausea and vomiting to be significantly distressing symptoms amongst patients undergoing chemotherapy for ovarian carcinoma. One reason for this can be attributed to the advent and usage of effective new antiemetic drugs, the efficacy of which can be seen in the far fewer reports of vomiting but with much less progress in the management of nausea (Reilly et al., 2013).
The pattern of symptoms reported in this study is similar to the symptom pattern of lung cancer patients on chemotherapy (Tchekmedyian et al., 2003; Akin et al., 2010). Lung cancer is associated with the highest levels of psychological distress relative to other types of cancer (Walling et al., 2014), possibly due to the physical symptoms of the disease. In addition, the frequency of symptoms in this study is much higher than other studies conducted on cancer patients using the MSAS-SF (Portenoy et al., 1994b; Lobchuk and Degner, 2002; Manitta et al., 2011). This is consistent with advanced stage ( $41.9 \%$ of patients in this study were stage IV), older age and being on treatment. Based on the high global distress index levels and corresponding psychological symptoms subscale however, this may also be due to greater psychological distress manifesting as psychosomatic symptoms. Differentiation of some of the most frequent symptoms such as fatigue and lack of appetite however remains an issue due to overlap over the anhedonic nature of such symptoms (Kroenke, Johns, Theobald et al., 2013).

These findings suggest that complaints of somatic symptoms such as fatigue and lack of appetite are related to psychological distress and should be further evaluated for clinically significant depression and/or anxiety using psychiatric interviews. Oncologists should therefore regard inexplicable symptoms or a higher frequency of reported symptoms as possibly attributable to psychological factors. This may also apply for symptoms that are seemingly inexplicable or of a reported degree severity that is inconsistent with disease or treatment side effects, or which persist despite adequate pharmacologic care and management.

This study additionally highlights the psychological aspect of cancer symptomology in patients undergoing chemotherapy. Because the primary focus on cancer is typically and foremost on its physical treatment, symptoms are almost always treated medically. Cancer patients and their oncologists thus often underestimate the psychological component underlying frequent or multiple reported symptoms and do not consider the potential benefit of using psychological treatments to help manage symptoms. Our findings are in line with previous research (Valentine and Meyers, 2001; Oh and So, 2010) indicating that psychological distress, rather than symptom distress, is likely to be more strongly related to symptoms such as fatigue. This suggests that psychological approaches are likely to be a useful means of management.

Some study limitations should be considered. First, as this is a cross-sectional study, it is not possible to infer causal conclusions. Symptoms and distress were measured from a single point in time, although results here are in line with those from longitudinal studies (Tchekmedyian et al., 2003). Serial measurement of symptoms and its associated distress will enable prospective assessment of how these two factors interrelate and change over time. The use of a generic scale, although well-validated and demonstrated to have sound psychometric characteristics, was not necessarily sensitive or specific to the measurement of psychological distress. Another limitation has to do with our patient sample, which was derived from a single 
academic medical centre, which limits more extensive generalisability of findings. Future studies that employ a cross-cultural perspective would prove interesting in investigating the differences in the expression of psychological distress and reported symptom frequency between Western and Asian countries.

In conclusion, There is a need for greater scrutiny in symptom assessment to allow the institution of intervention to control them. This study additionally allows for identification of symptom clusters that are more likely to be associated with distress. A higher frequency of reported symptoms than normal may actually indicate a subconscious bid for care and reassurance from their oncologists. Tailored multidisciplinary intervention which includes the management of psychological distress should be offered to patients reporting multiple symptoms in order to improve patient outcomes.

\section{Acknowledgements}

This work was supported by High Impact Research (HIR) grant number UM.C/HIR/MOHE/06/H-500100-A000020-000001 from University of Malaya.

\section{References}

Akechi T, Okuyama T, Endo C, et al (2011). Patient's perceived need and psychological distress and/or quality of life in ambulatory breast cancer patients in Japan. Psycho Oncol, 20, 497-505.

Akin S, Can G,Avdiner A, et al (2010). Quality of life, symptom experience and distress of lung cancer patients undergoing chemotherapy. Eur J Oncol Nurs, 14, 400-9.

Borjeson S, Starkhammar H, Unosson M, Bertero C (2012). Common symptoms and distress experienced among patients with colorectal cancer: a qualitative part of mixed method design. Open Nurs J, 6, 100-7.

Breen SJ, Baravelli CM, Schofield PE, et al (2009). Is symptom burden a predictor of anxiety and depression in patients with cancer about to commence chemotherapy? Med J Aust, 190, 99-104.

Chang VT, Huang SS, Feuerman M, et al (2000a). Symptom and quality of life survey of medical oncology patients at a veteran affairs medical centre: a role for symptom assessment. Cancer, 88, 1175-83.

Chang VT, Huang SS, Feuerman M, et al (2000b). Memorial symptoms assessment scale short form. Cancer, 89, 1162-71.

Cleeland CS (2007). Symptom burden: multiple symptoms and their impact as patient-reported outcomes. J Natl Cancer Inst Monogr, 37, 16-21.

Cooley ME, Short TH, Moriarty HJ, et al (2003). Symptom prevalence, distress and change over time in adults receiving treatment for lung cancer. Psycho Oncol, 12(7), 694-708.

Delgado-Guay M, Parsons HA, Li Z, et al (2009). Symptom distress in advanced cancer patients with anxiety and depression in the palliative care setting. Support Care Cancer, 17, 573-9.

Ferguson RJ, Ahles TA (2003). Low neuropsychologic performance among adult cancer survivors treated with chemotherapy. Curr Neurol Neurosci Rep, 3, 215-22.

Fitzgerald P, Lo C, Li M, Gagliese L, Zimmerman C, Rodin G (2013). The relationship between depression and physical symptom burden in advanced cancer. BMJ Support Palliat Care, 5, 381-8
Heyn L, Ruland CM, Finset A (2012). Effects of an interactive tailored assessment tool on eliciting and responding to cancer patients' cues and concerns in clinical consultations with physicians and nurses. Pat Educ Counsel, 86, 158-65.

Huijer HA, Abboud S, Doumit M (2012). Symptom prevalence and management of cancer patients in Lebanon. $J$ Pain Symptom Manage, 44, 386-99.

Illi J, Miaskowski C, Cooper B, et al (2012). Association between pro- and anti-inflammatory cytokine genes and a symptom cluster of pain, fatigue, sleep disturbance, and depression. Cytokine, 58, 437-47.

Kim JE, Dodd MJ, Aouizerat BE, et al (2009). A review of prevalence and impact of multiple symptoms in oncology patients. J Pain Symptom Manage, 37, 715-36.

Kroenke K, Johns SA, Theobald D, Wu J, Tu W (2013). Somatic symptoms in cancer patients trajectory over 12 months and impact on functional status and disability. Support Care Cancer, 21, 765-73.

Lobchuk MM, Degner LF (2002). Symptom experiences: perceptual accuracy between advanced stage cancer patients and family care-givers in the home care setting. JClin Oncol, 20, 3495-507.

Manitta V,Zordan R, Cole-Sinclair M, et al (2011). The symptom burden of patients with haematological malignancy: a crosssectional observational study. J Pain Symptom Manage, 42, 432-42.

Miaskowski C, Cooper BA, Melisko M, et al (2014). Disease and treatment characteristics do not predict symptom occurrence profiles in oncology outpatients receiving chemotherapy. Cancer, 120, 2371-8.

Oh HS, So WS (2010). Systematic review and meta-analysis of the correlates of cancer related-fatigue. Evidence Based Nursing, 191-201.

Petterson G, Bertero C, Unosson M, Borjeson S (2014). Symptom prevalence, frequency, severity, and distress during chemotherapy for patients with colorectal cancer. Support Care Cancer, 22, 1171-9.

Portenoy RK, Thaler HT, Kornblith AB, et al (1994a). Symptom prevalence, characteristics and distress in a cancer population. Qual Life Res, 3, 183-9.

Portenoy RK, Kornblith AB, Wong G, et al (1994b). Prevalance, characteristics and associated symptoms. Cancer, 74, 90715 .

Reilly CM, Bruner DW, Mitchell, et al (2013). A literature synthesis of symptom prevalence and severity in persons receiving active cancer treatment. Support Care Cancer, 21, 1525-50.

Rhodes VA, Watson PM (1987). Symptom distress - the concept: Past and present. Seminars Oncol Nurs, 3, 242-7.

Stasi R, Abriani L, Baccaglia P, et al (2003). Cancer-related fatigue: Evolving concepts in evaluation and treatment. Cancer, 98, 1786-801.

Sun CC, Bodurka DC, Weaver CB, et al (2005). Rankings and symptom assessment of side effects of chemotherapy: insight from experienced patients with ovarian cancer. Support Care Cancer, 13, 219-27.

Spichiger E, Muller-Frohlich C, Denhaerynck K, et al (2011). Prevalence of symptoms, with a focus on fatigue, and changes of symptoms over three months in outpatients receiving cancer chemotherapy. Swiss Medical Weekly, 141, 13303

Tchekmedyian NS, Kallich J, McDermott A, et al (2003). The relationship between psychologic distress and cancer related fatigue. Cancer, 98, 198-203.

Uitterhoeve R, Bensing J, Dilven E, et al (2009). Nurse-patient communication in cancer care: does responding to patient's cues predict patient satisfaction with communication. Psycho 
Muthukkumaran Thiagarajan et al

Oncol, 18, 1060-8.

Valentine AD, Meyers CA (2001). Cognitive and mood disturbances as causes and symptoms of fatigue in cancer patients. Cancer, 92, 1694-8.

Walling AM, Weeks JC, Kahn KL, et al (2014). Symptom prevalence in lung and colorectal cancer patients. J Pain Symptom Manage, 49, 192-202.

Yahaya NA, Subramaniam P, Bustam AZ, Taib NA (2015). Symptom experiences and coping strategies among multiethnic solid tumor patients undergoing chemotherapy in Malaysia, Asian Pac J Cancer Prev, 16, 723-30.

Zabora J, Brintzenhofe SK, Curbow B, et al (2001). The prevalence of psychological distress by cancer site. Psycho Oncol, 10, 19-28. 